\title{
Common fixed point of $g$-approximative multivalued mapping in ordered partial metric space
}

\section{Ali Erduran}

\section{Correspondence:}

ali.erduran1@yahoo.com

Department of Mathematics,

Faculty of Arts and Sciences,

Kirikkale University, Yahsihan,

Kirikkale 71450, Turkey

\begin{abstract}
In this paper, we introduce $g$-approximative multivalued mappings to a partial metric space. Based on this definition, we give some new definitions. Further, common fixed point results for $g$-approximative multivalued mappings satisfying generalized contractive conditions are obtained in the setup of ordered partial metric spaces.

MSC: Primary 05C38; 15A15; secondary 05A15; $15 \mathrm{~A} 18$

Keywords: $g$-approximative multivalued mapping; ordered partial metric space
\end{abstract}

\section{Introduction and preliminaries}

The study of fixed points for multivalued contraction mappings using the Hausdorff metric was initiated by Nadler [1]. After this, fixed point theory has been developed further and applied to many disciplines to solve functional equations. The Banach contraction principle has been extended in different directions either by using generalized contractions for multivalued mappings and hybrid pairs of single and multivalued mappings, or by using more general spaces. Dhage [2,3] established hybrid fixed point theorems and obtained some applications of presented results. Hong and Shen [4] proved common fixed point results for generalized contractive multivalued operators in a complete metric space. Also, the monotone iterative technique is associated with several nonlinear problems [5]. This technique is also employed to prove the existence of fixed points for multivalued monotone operators (see, for example, [6]). In [6], the problem of existence and approximation of coupled fixed points for mixed monotone multivalued operators was studied in ordered Banach spaces under the assumption that operators satisfy the condensing condition and upper demicontinuity.

Hong introduced the concepts of approximative values, comparable approximative values, upper and lower comparable approximative values in [7]. These definitions are a very useful tool for proving the existence of a fixed point of a multivalued operator in an ordered metric space. Then, Abbas and Erduran in [8] extended the concept of these definitions using $g$ self-mappings, so they introduced $g$-approximative multivalued mappings and proved coincidence and common fixed point results for a hybrid pair of multivalued and single-valued mappings. Also, they introduced the concepts of $g$-comparable approximative, $g$-upper comparable approximative and $g$-lower comparable approximative multivalued mappings.

2013 Erduran: licensee Springer This is an Open Access article distributed under the terms of the Creative Commons Attribution License (http://creativecommons.org/licenses/by/2.0), which permits unrestricted use, distribution, and reproduction in any medium, provided the original work is properly cited. 
In this paper, unless otherwise mentioned, let $(X, p)$ denote an ordered complete partial metric space with a partial order $\leq$ and distance $p(\cdot, \cdot)$.

Definition 1 Let $X$ be an ordered partial metric space. A mapping $g: X \rightarrow X$ is said to be (i) weakly $L$-idempotent if $g x \leq g^{2} x$ for $x$ in $X$, (ii) weakly $R$-idempotent if $g^{2} x \leq g x$ for $x$ in $X$. For example, a mapping $g:[0,1] \rightarrow[0,1]$ given by $g(x)=x^{2}$ is weakly $R$-idempotent.

Definition 2 An ordered partial metric space is said to have a subsequential limit comparison property if for every nondecreasing sequence (nonincreasing sequence) $\left\{x_{n}\right\}$ in $X$ such that $x_{n} \rightarrow x$, there exists a subsequence $\left\{x_{n_{k}}\right\}$ of $\left\{x_{n}\right\}$ with $x_{n_{k}} \leq x\left(x \leq x_{n_{k}}\right)$, respectively.

Definition 3 An ordered partial metric space is said to have a sequential limit comparison property if for every nondecreasing sequence (nonincreasing sequence) $\left\{x_{n}\right\}$ in $X$ such that $x_{n} \rightarrow x$ implies that $x_{n} \leq x\left(x \leq x_{n}\right)$, respectively.

Let $X$ be any nonempty set endowed with a partial order $\leq$ and let $g: X \rightarrow X$ be a given mapping. We define the set $\Delta_{g} \subseteq X \times X$ by

$$
\Delta_{g}=\{(x, y) \in X \times X: g x \leq g y\} .
$$

Note that for each $x \in X$, one has $(x, x) \in \Delta_{g}$.

Example 1 Let $X=\{0,1,2\}$ be endowed with the usual order $\leq$ and $g$ be a self-map on $X$ defined as $g 0=0, g 1=2$ and $g 2=1$. Then the subset $\Delta_{g}$ of $X \times X$ is $\Delta_{g}=$ $\{(0,0),(0,1),(0,2),(1,1),(2,1),(2,2)\}$.

In order to extend the concept of $g$-approximative, $g$-CAV, $g$-UCAV, $g$-LCAV multivalued mappings on partial metric spaces, we first adapt the notion of $g$-approximative to the partial metric framework as follows.

Definition 4 Let $X$ be a partial metric space and $g: X \rightarrow X$. A subset $Y$ of $X$ is said to be $g$-approximative for some $x$ in $X$ if $Y \subset g(X)$ and the set

$$
\Delta_{Y}^{g}(g(x))=\{y \in Y: p(g(x), y)=p(Y, g(x))\}
$$

is nonempty.

Definition 5 Let $X$ be a partially ordered set. A mapping $F: X \rightarrow 2^{X}$ (collection of all nonempty subsets of $X$ ) is said to be:

(i) $g$-approximative multivalued mapping (in short $g$-AV multivalued mapping), if $F x$ is $g$-approximative for each $x \in X$, that is, $\Delta_{F x}^{g}(g(x))$ is nonempty for each $x$ in $X$.

(ii) $g$-CAV multivalued mapping ( $g$-comparable approximative multivalued mapping) if $F$ is $g$-approximative and for each $z \in X$, there exists $g(y) \in \Delta_{F(z)}^{g}(g(z))$ such that $g y$ is comparable to $g z$.

(iii) $g$-UCAV ( $g$-upper comparable approximative multivalued mapping) if $F$ is $g$-approximative and for each $z \in X$, there exists $g(y) \in \Delta_{F(z)}^{g}(g(z))$ such that $g(z) \leq g(y)$. 
(iv) $g$-LCAV ( $g$-lower comparable approximative multivalued mapping) if $F$ is $g$-approximative and for each $z \in X$, there exists $g(y) \in \Delta_{F(z)}^{g}(g(z))$ such that $g(y) \leq g(z)$.

If $F$ is single-valued, then $g$-UCAV $(g$-LCAV) means that $F x \geq g x(F x \leq g x)$ for $x \in X$.

Definition 6 Let $g: X \longrightarrow X$ and $T: X \longrightarrow C B(X)$. A point $x$ in $X$ is said to be: (i) a fixed point of $g$ if $g(x)=x$, (ii) a fixed point of $T$ if $x \in T(x)$, (iii) a coincidence point of a pair $(g, T)$ if $g x \in T x$, (iv) a common fixed point of a pair $(g, T)$ if $x=g x \in T x$.

$F(g), C(g, T)$ and $F(g, T)$ denote the set of all fixed points of $g$, the set of all coincidence points of the pair $(g, T)$ and the set of all common fixed points of the pair $(g, T)$, respectively.

Definition 7 Let $f: X \longrightarrow X, T: X \longrightarrow C B(X)$ and $f T x \in C B(X)$. The pair $(f, T)$ is called (1) commuting if $T f x=f T x$ for all $x \in X$, (2) weakly compatible [9] if they commute at their coincidence points, that is, $f T x=T f x$ whenever $x \in C(f, T)$, (3) (IT)-commuting at $x \in X$ if $f T x \subseteq T f x$.

Definition 8 Let $T: X \longrightarrow C B(X)$. The map $f: X \longrightarrow X$ is said to be $T$-weakly commuting at $x \in X$ if $f^{2} x \in T f x$.

Definition 9 The map $f: X \rightarrow X$ is said to be coincidently idempotent with respect to $T: X \rightarrow C B(X)$ if $f^{2}(x)=f(x)$ for $x$ in $C(f, T)$. The point $x$ is called a point of coincident idempotency.

Now, we present an example of a hybrid pair $\{f, T\}$ for which $f$ is $T$-weakly commuting at some $x \in C(f, T)$.

Example 2 Let $X=[0, \infty)$ with the usual metric. Define $f: X \rightarrow X, T: X \rightarrow C B(X)$ by

$$
f x= \begin{cases}0, & 0 \leq x<1 \\ x+1, & 1 \leq x<\infty\end{cases}
$$

and

$$
T x= \begin{cases}\{x\}, & 0 \leq x<1 \\ {[1, x+2],} & 1 \leq x<\infty\end{cases}
$$

It can be easily verified that $f$ is $T$-weakly commuting at $x=0 \in C(f, T)$.

Example 3 Let $X=R$ with the usual metric. Define $f: X \rightarrow X, T: X \rightarrow C B(X)$ by

$$
f x= \begin{cases}-1, & x \leq 0, \\ -\frac{2}{x}, & 0<x\end{cases}
$$


and

$$
T x= \begin{cases}\{x\}, & x \leq-1 \\ {[x, 1],} & -1<x \leq 1 \\ {[1, x],} & 1<x<\infty\end{cases}
$$

Here $C(f, T)=\{-1\}$ and $f$ is coincidently idempotent with respect to $T$.

Let $\alpha \in(0,+\infty]$. $\digamma$ denotes the class of mappings $f:[0, \alpha) \rightarrow \mathbb{R}$ which satisfy the following conditions:

(i) $f(0)=0$ and $f(t)>0$ for each $t \in(0, \alpha)$,

(ii) $f$ is continuous,

(iii) $f$ is nondecreasing on $[0, \alpha)$.

A mapping $f$ is said to be sublinear if $f\left(t_{1}+t_{2}\right) \leq f\left(t_{1}\right)+f\left(t_{2}\right)$ whenever $t_{1}, t_{2}, t_{1}+t_{2} \in(0, \alpha)$. We define $\digamma_{s}=\{f:[0, \alpha) \rightarrow \mathbb{R}: f$ is sublinear and $f \in \digamma\}$.

$\Psi$ denotes the family of mappings $\psi:[0, \alpha) \rightarrow[0,+\infty)$ which satisfy the following conditions:

(a) $\psi(t)<t$ for each $t \in(0, \alpha)$,

(b) $\psi$ is nondecreasing and right upper semi-continuous,

(c) for each $t \in(0, \alpha), \lim _{n \rightarrow \infty} \psi^{n}(t)=0$.

By means of the functions $f$ and $\psi$ given in $\digamma$ and $\psi$ respectively, a generalized contractive condition was defined in [10]. Let $\Phi$ denote the class of mappings $\psi:[0, \alpha) \rightarrow[0,+\infty)$ for which $\psi(t)<t$ and $\sum_{n=1}^{\infty} \psi^{n}(t)<\infty$ for each $t$ in $(0, \alpha)$.

Definition 10 For two subsets $A, B$ of $X$, we say $A \leq_{1} B$ if for each $x \in X$, there exists $y \in Y$ such that $x \leq y$ and $A \leq B$ if each $x \in A, y \in B$ implies that $x \leq y$.

A multivalued mapping $F: X \rightarrow 2^{X}$ is said to be $g$-nondecreasing ( $g$-nonincreasing) if $g x \leq g y$ implies that $F x \leq_{1} F y\left(F y \leq_{1} F x\right)$ for all $x, y \in X$. $F$ is said to be $g$-monotone if $F$ is $g$-nondecreasing or $g$-nonincreasing. Moreover, in what follows, $(X, \leq)$ will be a partially ordered set such that there exists a complete partial metric $p$ on $X$. Let $D=\sup \{p(x, y)$ : $x, y \in X\}$. Set $\alpha=p$ if $p=\infty$ and $\alpha>p$ if $p<\infty$.

Consistent with [11-13], the following definitions and results will be needed in the sequel.

Definition 11 A partial metric on a nonempty set $X$ is a function $p: X \times X \rightarrow R^{+}$such that for all $x, y, z \in X$,

(i) $x=y \Leftrightarrow p(x, x)=p(x, y)=p(y, y)$,

(ii) $p(x, x) \leq p(x, y)$,

(iii) $p(x, y)=p(y, x)$,

(iv) $p(x, y) \leq p(x, z)+p(z, y)-p(z, z)$.

A partial metric space is a pair $(X, p)$ such that $X$ is a nonempty set and $p$ is a partial metric on $X$.

Each partial metric $p$ on $X$ generates a $T_{0}$ topology $\tau_{p}$ on $X$ which has as a base the family of open $p$-balls $\left\{B_{p}(x, \varepsilon), x \in X, \varepsilon>0\right\}$, where $B_{p}(x, \varepsilon)=\{y \in X: p(x, y)<p(x, x)+\varepsilon\}$ for all $x \in X$ and $\varepsilon>0$. 
If $p$ is a partial metric on $X$, then the function $p^{s}: X \times X \rightarrow R^{+}$given by

$$
p^{s}(x, y)=2 p(x, y)-p(x, x)-p(y, y)
$$

is a metric on $X$.

A mapping $f: X \rightarrow X$ is said to be continuous at $x_{0} \in X$ if for every $\varepsilon>0$, there exists $\delta>0$ such that $f\left(B_{p}\left(x_{0}, \delta\right)\right) \subset B_{p}\left(f\left(x_{0}\right), \varepsilon\right)$.

Definition 12 Let $(X, p)$ be a partial metric space and $\left\{x_{n}\right\}$ be a sequence in $X$. Then

(i) $\left\{x_{n}\right\}$ converges to a point $x \in X$ if and only if $p(x, x)=\lim _{n \rightarrow+\infty} p\left(x, x_{n}\right)$,

(ii) $\left\{x_{n}\right\}$ is called a Cauchy sequence if there exists (and is finite) $\lim _{n, m \rightarrow+\infty} p\left(x_{n}, x_{m}\right)$.

Definition 13 A partial metric space $(X, p)$ is said to be complete if every Cauchy sequence $\left\{x_{n}\right\}$ in $X$ converges, with respect to $\tau_{p}$, to a point $x \in X$ such that $p(x, x)=p\left(x_{n}, x_{m}\right)$.

Lemma 1 Let $(X, p)$ be a partial metric space. Then

(a) $\left\{x_{n}\right\}$ is a Cauchy sequence in $(X, p)$ if and only if it is a Cauchy sequence in the metric space $\left(X, p_{s}\right)$,

(b) $(X, p)$ is complete if and only if the metric space $\left(X, p^{s}\right)$ is complete. Furthermore, $\lim _{n \rightarrow+\infty} p^{s}\left(x_{n}, x\right)=0$ if and only if

$$
p(x, x)=\lim _{n \rightarrow+\infty} p\left(x_{n}, x\right)=\lim _{n, m \rightarrow+\infty} p\left(x_{n}, x_{m}\right) .
$$

Lemma 2 Let $(X, p)$ be a partial metric space and let $T: X \rightarrow X$ be a continuous selfmapping. Assume $\left\{x_{n}\right\} \in X$ such that $x_{n} \rightarrow z$ as $n \rightarrow \infty$. Then

$$
\lim _{n \rightarrow \infty} p\left(T x_{n}, T z\right)=p(T z, T z)
$$

Recently Haydi et al. introduced a partial Hausdorff metric on a partial metric space and they extended Nadler's fixed point theorem on partial metric spaces using the partial Hausdorff metric.

Let $(X, p)$ be a partial metric space. Let $C B^{p}(X)$ be a family of all nonempty, closed and bounded subsets of the partial metric space $(X, p)$, induced by the partial metric $p$. Note that closedness is taken from $\left(X, \tau_{p}\right)\left(\tau_{p}\right.$ is the topology induced by $\left.p\right)$ and boundedness is given as follows: $\mathrm{A}$ is a bounded subset in $(X, p)$ if there exist $x_{0} \in X$ and $M \geq 0$ such that for all $a \in A$, we have $a \in B_{p}\left(x_{0}, M\right)$, that is, $p\left(x_{0}, a\right)<p(a, a)+M$.

For $A, B \in C B^{p}(X)$ and $x \in X$,

$$
\begin{aligned}
& p(x, A)=\inf \{p(x, y): y \in A\}, \\
& \delta_{p}(A, B)=\sup \{p(a, B): a \in A\}, \\
& \delta_{p}(B, A)=\sup \{p(A, b): b \in B\} .
\end{aligned}
$$

It is immediate to check that $p(x, A)=0 \Rightarrow p^{s}(x, A)=0$, where $p^{s}(x, A)=\inf \left\{p^{s}(x, a): a \in\right.$ $A\}$. 
Remark 1 ([14]) Let $(X, p)$ be a partial metric space and $A$ be any nonempty set in $(X, p)$, then $a \in \bar{A}$ if and only if $p(a, A)=p(a, a)$, where $A$ denotes the closure of $A$ with respect to the partial metric $p$. Note that $A$ is closed in $(X, p)$ if and only if $\bar{A}=A$.

Proposition $1([14])$ Let $(X, p)$ be a partial metric space. For any $A, B, C \in C B^{p}(X)$, we have the following:

(i) $\delta_{p}(A, A)=\sup \{p(a, a): a \in A\}$;

(ii) $\delta_{p}(A, A) \leq \delta_{p}(A, B)$;

(iii) $\delta_{p}(A, B)=0$ implies that $A \subset B$;

(iv) $\delta_{p}(A, B) \leq \delta_{p}(A, C)+\delta_{p}(C, B)-\inf _{c \in C} p(c, c)$.

Proposition 2 ([14]) Let $(X, p)$ be a partial metric space. For all $A, B, C \in C B^{p}(X)$, we have

(h1) $H_{p}(A, A) \leq H_{p}(A, B)$;

(h2) $H_{p}(A, B)=H_{p}(B, A)$;

(h3) $H_{p}(A, B) \leq H_{p}(A, C)+H_{p}(C, B)-\inf _{c \in C} p(c, c)$.

Note that $(X, p)$ is a partial metric space. For $A, B \in C B^{p}(X)$, the following holds: $H_{p}(A, B)=0$ implies that $A=B$. The converse of this case is not true in general as it is clear from the following example.

Example 4 ([14] $)$ Let $X=[0,1]$ be endowed with the partial metric $p: X \times X \rightarrow R^{+}$defined by $p(x, y)=\max \{x, y\}$. From (i) of Proposition 2.2, we have

$$
H_{p}(X, X)=\delta_{p}(X, X)=\sup \{x: 0 \leq x \leq 1\}=1 \neq 0 .
$$

In view of Proposition 2.3 and Corollary 2.4 in [14], we call the mapping $H_{p}: C B^{p}(X) \times$ $C B^{p}(X) \rightarrow[0,+\infty)$ a partial Hausdorff metric induced by $p$.

Remark 2 It is easy to show that any Hausdorff metric is a partial Hausdorff metric. The converse is not true (Example 2.6 of in [14]).

The aim of this paper is to adapt the notion of $g$-approximative to the partial metric and extend the concept of $g$-UCAV, $g$-LCAV, $g$-CAV mappings. Also, we prove some fixed point theorems for multivalued mappings and give an example associated with the following theorem.

Theorem 1 Suppose that $g$ is a nondecreasing self-map on $X$ and $F: X \rightarrow 2^{X}$ is $g$-UCAV and the following holds:

$$
f\left(H_{p}(F x, F y)\right) \leq \psi\left(f\left(M_{g}(x, y)\right)\right)
$$

for any $(x, y) \in \Delta_{g}$, where $f \in \digamma_{s}$ and $\psi \in \Phi$ and

$$
M_{g}(x, y)=\max \left\{p(g x, g y), p(g x, F x), p(g y, F y), \frac{p(g x, F y)+p(g y, F x)}{2}\right\} .
$$

If $X$ has the limit comparison property and $g(X)$ is closed, then $F$ and $g$ have a coincidence point $x$ in $X$. Moreover, $F$ and $g$ have a common fixed point if one of the following conditions holds: 
(i) Pair $(F, g)$ is IT-commuting at some $x \in C(F, g)$ and $\lim _{n \rightarrow \infty} g^{n} x=u$ for some $u \in X$ and $g$ is continuous at $u$.

(ii) Pair $(F, g)$ is IT-commuting at some $x \in C(F, g)$ and $g^{2} x=g x$.

(iii) $g$ is $F$-weakly commuting at some $C(F, g)$ and $g$ is coincidently idempotent with respect to $T$.

(iv) $g$ is continuous at $x$ for some $x \in C(F, g)$ for some $u \in X$; $\lim g^{n} u=x$.

(v) $g(C(g, F))$ is a singleton subset of $C(g, F)$.

Proof Let $x_{0} \in X$. If $g x_{0} \in F x_{0}$, then the result is proved. If not, then we proceed as follows. As $F$ is $g$-UCAV, $F x_{0} \subset g(X), \Delta_{F\left(x_{0}\right)}^{g}\left(g\left(x_{0}\right)\right)$ is nonempty, so there exists $g x_{1} \in F x_{0}$ with $g x_{1} \neq$ $g x_{0}$ such that $p\left(g x_{1}, g x_{0}\right)=p\left(F x_{0}, g x_{0}\right)$ for some $x_{1} \in X$ and $g x_{1} \geq g x_{0}$. Similarly, there exists $g x_{2} \in F x_{1}$ with $g x_{1} \neq g x_{2}$ such that $p\left(g x_{1}, g x_{2}\right)=p\left(F x_{1}, g x_{1}\right)$ for some $x_{2} \in X$, and $g x_{2} \geq g x_{1}$. We continue to construct a sequence $\left\{x_{n}\right\}$ for which either $g x_{n-1} \in F x_{n-1}$ or there exists $g x_{n} \in F x_{n-1}$ with $g x_{n} \neq g x_{n-1}$ and $g x_{n} \geq g x_{n-1}$ such that

$$
p\left(g x_{n}, g x_{n-1}\right)=p\left(F x_{n-1}, g x_{n-1}\right), \quad \text { for } n=1,2, \ldots
$$

for some $x_{n}$ in $X$. On the other hand,

$$
p\left(F x_{n-1}, g x_{n-1}\right)=\sup _{x \in F x_{n-2}} p\left(x, F x_{n-1}\right) \leq H_{p}\left(F x_{n-1}, F x_{n-2}\right)
$$

implies that

$$
p\left(g x_{n}, g x_{n-1}\right) \leq H_{p}\left(F x_{n-1}, F x_{n-2}\right), \quad \text { for } n=2,3, \ldots
$$

Since $f$ is nondecreasing, we have

$$
\begin{aligned}
f\left(p\left(g x_{n}, g x_{n-1}\right)\right) & \leq f\left(H_{p}\left(F x_{n-1}, F x_{n-2}\right)\right) \\
& \leq \psi\left(f\left(M_{g}\left(x_{n-1}, x_{n-2}\right)\right)\right)
\end{aligned}
$$

in which

$$
\begin{aligned}
M_{g}\left(x_{n-1}, x_{n-2}\right) & \max \left\{p\left(g x_{n-1}, g x_{n-2}\right), p\left(F x_{n-1}, g x_{n-1}\right), p\left(F x_{n-2}, g x_{n-2}\right),\right. \\
& \left.\frac{p\left(F x_{n-2}, g x_{n-1}\right)+p\left(F x_{n-1}, g x_{n-2}\right)}{2}\right\} \\
= & \max \left\{p\left(g x_{n-1}, g x_{n-2}\right), p\left(g x_{n}, g x_{n-1}\right), p\left(g x_{n-1}, g x_{n-2}\right),\right. \\
& \left.\frac{p\left(g x_{n-1}, g x_{n-1}\right)+p\left(g x_{n}, g x_{n-2}\right)}{2}\right\} \\
= & \max \left\{p\left(g x_{n-1}, g x_{n-2}\right), p\left(g x_{n}, g x_{n-1}\right)\right\} .
\end{aligned}
$$


If $p\left(g x_{n-1}, g x_{n}\right) \geq p\left(g x_{n-1}, g x_{n-2}\right)$, then we have

$$
\begin{aligned}
f\left(p\left(g x_{n}, g x_{n-1}\right)\right) & \leq f\left(H_{p}\left(F x_{n-1}, F x_{n-2}\right)\right) \\
& \leq \psi\left(f\left(M_{g}\left(x_{n-1}, x_{n-2}\right)\right)\right) \\
& \leq \psi\left(f\left(\max \left\{p\left(g x_{n-1}, g x_{n-2}\right), p\left(g x_{n-1}, g x_{n}\right)\right\}\right)\right) \\
& \leq \psi\left(f\left(p\left(g x_{n}, g x_{n-1}\right)\right)\right) \\
& <f\left(p\left(g x_{n}, g x_{n-1}\right)\right)
\end{aligned}
$$

a contradiction. So, we have $p\left(g x_{n-1}, g x_{n-2}\right)>p\left(g x_{n-1}, g x_{n}\right)$. This yields

$$
f\left(p\left(g x_{n}, g x_{n-1}\right)\right) \leq \psi\left(f\left(p\left(g x_{n-1}, g x_{n-2}\right)\right)\right) .
$$

Repeating this process, we have

$$
\begin{aligned}
f\left(p\left(g x_{n}, g x_{n-1}\right)\right) \leq & \psi\left(f\left(p\left(g x_{n-1}, g x_{n-2}\right)\right)\right) \\
\leq & \psi^{2}\left(f\left(p\left(g x_{n-2}, g x_{n-3}\right)\right)\right) \\
& \vdots \\
\leq & \psi^{n-1}\left(f\left(p\left(g x_{0}, g x_{1}\right)\right)\right) .
\end{aligned}
$$

For $m, n \in \mathbb{N}$, we obtain

$$
p\left(g x_{n}, g x_{n+m}\right) \leq \sum_{i=n}^{n+m-1} p\left(x_{i}, x_{i+1}\right) .
$$

This implies

$$
\begin{aligned}
f\left(p\left(g x_{n}, g x_{n+m}\right)\right) & \leq f\left(p\left(g x_{n}, g x_{n+1}\right)+\cdots+p\left(g x_{n+m-1}, g x_{n+m}\right)\right) \\
& \leq f\left(p\left(g x_{n}, g x_{n+1}\right)\right)+\cdots+f\left(p\left(g x_{n+m-1}, g x_{n+m}\right)\right) \\
& \leq \psi^{n}\left(f\left(p\left(g x_{0}, g x_{1}\right)\right)\right)+\cdots+\psi^{n+m}\left(f\left(p\left(g x_{0}, g x_{1}\right)\right)\right) \\
& \leq \sum_{i=n}^{n+m-1} \psi^{i}\left(f\left(p\left(g x_{0}, g x_{1}\right)\right)\right) .
\end{aligned}
$$

On taking limit as $n \rightarrow \infty$ and using $\sum_{n=1}^{\infty} \psi^{n}(t)<\infty$, we have $\lim _{n \rightarrow \infty} p\left(g x_{n}, g x_{n+m}\right)=0$. By the definition of $p^{s}$, we get

$$
p^{s}\left(g x_{n}, g x_{n+m}\right) \leq 2 p\left(g x_{n}, g x_{n+m}\right) \rightarrow 0 \quad \text { as } n \rightarrow \infty .
$$

This yields that $\left\{g\left(x_{n}\right)\right\}$ is a Cauchy sequence in $\left(g(X), p^{s}\right)$. Since $X$ is complete and $g(X)$ is closed, then $(g(X), p)$ is complete, hence $\left(g(X), p^{s}\right)$ is complete. So, we have $\lim _{n \rightarrow \infty} p^{s}\left(g x_{n}, g x\right)=0$ for some $x \in X$. From Lemma 1, we get

$$
p(g x, g x)=\lim _{n \rightarrow \infty} p\left(g x_{n}, g x\right)=\lim _{n \rightarrow \infty} p\left(g x_{n}, g x_{m}\right)=0 .
$$


Consequently, $\left\{g\left(x_{n}\right)\right\}$ is a Cauchy sequence in $(g(X), p)$. Now, we prove that $p(F x, g x)=0$. Suppose that this is not true, then $p(F x, g x)>0$. For large enough $n$, we claim that the following equation holds:

$$
\begin{aligned}
M_{g}\left(x, x_{n+1}\right) & =\max \left\{p\left(g x, g x_{n+1}\right), p(F x, g x), p\left(F x_{n+1}, g x_{n+1}\right), \frac{p\left(F x, g x_{n+1}\right)+p\left(F x_{n+1}, g x\right)}{2}\right\} \\
& =p(F x, g x) .
\end{aligned}
$$

Indeed, since $\lim _{n \rightarrow \infty} p\left(g x_{n}, g x\right)=0$ and $\lim _{n \rightarrow \infty} p\left(F x_{n+1}, g x_{n+1}\right)=0$, it follows that

$$
\begin{aligned}
\lim _{n \rightarrow \infty} & \frac{1}{2}\left[p\left(F x, g x_{n+1}\right)+p\left(F x_{n+1}, g x\right)\right] \\
\quad \leq & \lim _{n \rightarrow \infty} \frac{1}{2}\left[p(F x, g x)+p\left(g x, g x_{n+1}\right)+p\left(F x_{n+1}, g x_{n+1}\right)+p\left(g x_{n+1}, g x\right)\right] \\
\quad= & \frac{1}{2} p(F x, g x) .
\end{aligned}
$$

So, there exists $n_{0} \in \mathbb{N}$ such that $M_{g}\left(x, x_{n+1}\right)=d(F x, g x)$ for every $n>n_{0}$. Note that

$$
f\left(p\left(F x, g x_{n+2}\right)\right) \leq f\left(H\left(F x, F x_{n+1}\right)\right) \leq \psi\left(f\left(M_{g}\left(x, x_{n+1}\right)\right)\right),
$$

which, on taking limit as $n \rightarrow \infty$, gives

$$
f(p(F x, g x)) \leq \psi(f(p(F x, g x)))<f(p(F x, g x))
$$

a contradiction. Hence, $p(F x, g x)=0$ and so $g x \in F x$. Suppose now that (i) holds. Then $\lim _{n \rightarrow \infty} g^{n} x=u$, where $u \in X$. Since $g$ is continuous at $u$, so we have that $u$ is a fixed point of $g$. By given assumption, $g^{n} x \in C\left(F, g^{n-1}\right)$ for all $n \geq 1$ and $g^{n} x \in F\left(g^{n-1} x\right)$. Now, we prove that $p(F u, g u)=0$. Suppose that this is not true, then $p(F u, g u)>0$. Using (1.1), since $f$ is nondecreasing and sublinear, we obtain

$$
\begin{aligned}
f(p(g u, F u)) & \leq f\left(p\left(g u, g^{n} x\right)\right)+f\left(p\left(g^{n} x, F u\right)\right) \\
& \leq f\left(p\left(g u, g^{n} x\right)\right)+f\left(H\left(F\left(g^{n-1} x\right), F(u)\right)\right) \\
& \leq f\left(p\left(g u, g^{n} x\right)\right)+\psi\left(f\left(M_{g}\left(g^{n-1} x, u\right)\right)\right),
\end{aligned}
$$

where

$$
\begin{aligned}
& M_{g}\left(g^{n-1} x, u\right) \\
& \quad=\max \left\{p\left(g^{n} x, g u\right), p\left(F g^{n-1} x, g^{n} x\right), p(F u, g u), \frac{p\left(F u, g^{n} x\right)+p\left(F g^{n-1} x, g u\right)}{2}\right\} \\
& \quad=\max \left\{p\left(g^{n} x, g u\right), p\left(g^{n} x, g^{n} x\right), p(F u, g u), \frac{p\left(F u, g^{n} x\right)+p\left(g^{n} x, g u\right)}{2}\right\} .
\end{aligned}
$$

On taking limit as $n \rightarrow \infty$, we have

$$
M_{g}\left(g^{n-1} x, u\right)=p(F u, g u)
$$


which further implies

$$
\begin{aligned}
f(p(g u, F u)) & \leq f\left(p\left(g u, g^{n} x\right)\right)+\psi(f(p(F u, g u))) \\
& <f\left(p\left(g u, g^{n} x\right)\right)+f(p(F u, g u)) .
\end{aligned}
$$

On taking limit as $n \rightarrow \infty$,

$$
f(p(g u, F u))<f(p(F u, g u))
$$

a contradiction, so $d(g u, F u)=0$ and hence $g u \in F u$. Consequently, $u=g u \in F u$. Hence, $u$ is a common fixed point of $F$ and $g$. Suppose now that (ii) holds. As $x \in C(F, g)$, so $g^{2} x \in g F x \subset F g x$. Now, $g x=g^{2} x \in F g x$ implies that $g x$ is a common fixed point of $F$ and $g$. Suppose now that (iii) holds. The result is obvious. Suppose that (iv) holds. As $x \in C(g, F)$ and for some $u \in X, \lim _{n \rightarrow \infty} g^{n} u=x$. By the continuity of $g$ at $x$, we get $x=g x \in F x$. Hence, $x$ is a common fixed point of $F$ and $g$. Finally, suppose that (v) holds. Let $g(C(F, g))=\{x\}$. Then $\{x\}=\{g x\}=F x$. Hence, $x$ is a common fixed point of $F$ and $g$.

Similarly, we have following theorem.

Theorem 2 Suppose that $g$ is a nondecreasing self-map on $X$ and $F: X \rightarrow 2^{X}$ is $g$-LCAV and the following holds:

$$
f\left(H_{p}(F x, F y)\right) \leq \psi\left(f\left(M_{g}(x, y)\right)\right)
$$

for any $(x, y) \in \Delta_{g}$, where $f \in \digamma_{s}$ and $\psi \in \Phi$ and

$$
M_{g}(x, y)=\max \left\{d(g x, g y), d(g x, F x), d(g y, F y), \frac{d(g x, F y)+d(g y, F x)}{2}\right\} .
$$

If $X$ has the sequential limit comparison property and $g(X)$ is closed, then $F$ and $g$ have a coincidence point $x$ in $X$. Moreover, $F$ and $g$ have a common fixed point if any one of the conditions (i)-(v) holds as in Theorem 1.

Example 5 Let $X=\{0\} \cup[1, \infty)$ with $p(x, y)=\max \{x, y\}$. Define $g: X \rightarrow X, F: X \rightarrow 2^{X}$ by

$$
g x= \begin{cases}0, & x=0 \\ x+1, & 1 \leq x<\infty\end{cases}
$$

and

$$
F x= \begin{cases}\{x\}, & x=0, \\ {[1, x+2],} & 1 \leq x<\infty .\end{cases}
$$

It is clear that $F$ is $g$-UCAV, also $g(X)$ is closed and $X$ has the property of limit comparison. We can see easily that $g$ is $F$-weakly commuting at $x=0$. Besides, $g$ is coincidently idempotent with respect to $F$ at $x=0$. In this case, these functions satisfy the condition of 
(iii) in Theorem 1. Also, we can define $f(t)=t, \psi(t)=\frac{t}{2}$, then $f \in \digamma_{s}$ and $\psi \in \Psi$. If $x=y=0$, we have $g x=g y=0$ and $F x=\{x\}, F y=\{y\}$

$$
\begin{aligned}
f\left(H_{p}(F x, F y)\right) & =H_{p}(F x, F y)=\max \left\{\sup _{z \in\{x\}} p(z,\{y\}), \sup _{t \in\{y\}}\{p(\{x\}, t)\}\right\} \\
& =\max \left\{\sup _{z \in\{0\}} \inf _{t \in\{0\}} p(z, t), \sup _{p \in\{0\}} \inf _{k \in\{0\}} p(p, k)\right\}=0 \\
& =\frac{\max \left\{p(g x, g y), p(g x, F x), p(g y, F y), \frac{p(g x, F y)+p(g y, F x)}{2}\right\}}{2} \\
& =\psi\left(f\left(M_{g}(x, y)\right)\right) .
\end{aligned}
$$

If $x=0, y \in[1, \infty)$, we have $g x=0, g y=y+1$ and $F x=\{x\}, F y=[1, y+2]$

$$
\begin{aligned}
f\left(H_{p}(F x, F y)\right) & =H_{p}(F x, F y)=\max \left\{\sup _{z \in\{0\}} d(z,[1, y+2]), \sup _{t \in[1, y+2]} d(\{0\}, t)\right\} \\
& =1 .
\end{aligned}
$$

Also, we have

$$
M_{g}(x, y)=\max \left\{y+1,0, \frac{y+2}{2}\right\}=y+1 .
$$

So, we satisfy the contractive condition. Finally, if $x, y \in[1, \infty)$, we have $g x=x+1, g y=y+1$ and $F x=[1, x+2], F y=[1, y+2]$

$$
\begin{aligned}
f\left(H_{p}(F x, F y)\right) & =H_{p}(F x, F y) \\
& =\frac{\max \left\{p(g x, g y), p(g x, F x), p(g y, F y), \frac{p(g x, F y)+p(g y, F x)}{2}\right\}}{2} \\
& \leq \psi\left(f\left(M_{g}(x, y)\right)\right) .
\end{aligned}
$$

Hence, all the conditions of Theorem 1 are satisfied. It is clear that $0=x=g x \in F x$, that is, $x=0$ is a common fixed point of $F$ and $g$.

Corollary 1 Suppose that $g$ is a nondecreasing self-map on $X$ and $F: X \rightarrow X$ and $g: X \rightarrow X$ are self-mappings which satisfy

$$
f(p(F x, F y)) \leq \psi\left(f\left(M_{g}(x, y)\right)\right)
$$

for any $(x, y) \in \Delta_{g}$, where $f \in \digamma_{s}, \psi \in \Phi$ and

$$
M_{g}(x, y)=\max \left\{p(g x, g y), p(F x, g x), p(F y, g y), \frac{p(F x, g y)+p(F y, g x)}{2}\right\} .
$$

Then $F, g$ have a unique coincidence point $x \in X$. Moreover, $F$ and $g$ have a unique common fixed point if any one of the conditions (i)-(v) holds as in Theorem 1. 
Proof Theorem 1 ensures the existence of a coincidence point. To prove the uniqueness, let $y$ be another coincidence point of $F$ and $g$. If $x \neq y$, then $p(g x, g y)>0$. Thus,

$$
M_{g}(x, y)=\max \left\{p(g x, g y), p(F x, g x), p(F y, g y), \frac{p(F x, g y)+p(F y, g x)}{2}\right\}=p(g x, g y) .
$$

This yields

$$
\begin{aligned}
f(p(g x, g y)) & =f(p(F x, F y)) \leq \psi\left(f\left(M_{g}(x, y)\right)\right) \\
& =\psi(f(p(g x, g y))) \\
& <f(p(g x, g y)),
\end{aligned}
$$

a contradiction, therefore $p(g x, g y)=0$. The result follows.

Theorem 3 Suppose that $g$ is a nondecreasing self-map on $X$ and $F: X \rightarrow 2^{X}$ is $g$-AV and the following holds:

$$
f\left(H_{p}(F x, F y)\right) \leq \psi\left(f\left(M_{g}(x, y)\right)\right)
$$

for any $(x, y) \in \Delta_{g}$, where $f \in \digamma_{s}$ and $\psi \in \Phi$ and

$$
M_{g}(x, y)=\max \left\{p(g x, g y), p(g x, F x), p(g y, F y), \frac{p(g x, F y)+p(g y, F x)}{2}\right\} .
$$

If $g(X)$ is closed and there exists $x_{0} \in X$ such that $\left\{g x_{0}\right\} \leq F x_{0}$, then $F$ and $g$ have a coincidence point $x \in X$. Further, an iterative sequence $\left\{g x_{n}\right\}$ with $g x_{n} \in F x_{n-1}$ converges to $g x$, where $x \in C(F, g)$. Moreover, $F$ and $g$ have a common fixed point if any one of the conditions (i)-(v) holds as in Theorem 1.

Proof If $g x_{0} \in F x_{0}$, then the proof is finished. Otherwise, for any $g x \in F x_{0}$, one has $g x \geq$ $g x_{0}$. As $F$ has a $g$-approximative multivalued map, for $x_{1} \in X$, there exists $g x_{1} \in F x_{0}$ with $g x_{1} \geq g x_{0}$ and

$$
p\left(g x_{0}, g x_{1}\right)=p\left(F x_{0}, g x_{0}\right) .
$$

Similarly, for $x_{2} \in X$, there exists $g x_{2} \in F x_{1}$ with $g x_{2} \geq g x_{1}$ and

$$
p\left(g x_{1}, g x_{2}\right)=p\left(F x_{1}, g x_{1}\right) .
$$

We continue the process of constructing a sequence $\left\{g x_{n}\right\}$ such that for $x_{n} \in X$, one obtains $g x_{n} \in F x_{n-1}$ with $g x_{n} \geq g x_{n-1}$ such that

$$
p\left(g x_{n-1}, g x_{n}\right)=p\left(F x_{n-1}, g x_{n-1}\right), \quad n=1,2, \ldots
$$

On the other hand, we have

$$
p\left(F x_{n-1}, g x_{n-1}\right)=\sup _{x \in F x_{n-2}} p\left(x, F x_{n-1}\right) \leq H_{p}\left(F x_{n-2}, F x_{n-1}\right) .
$$


So,

$$
p\left(g x_{n-1}, g x_{n}\right) \leq H_{p}\left(F x_{n-2}, F x_{n-1}\right) \quad \text { for } n=2,3, \ldots
$$

The rest of this proof is the same as that of Theorem 1.

Theorem 4 Suppose that $g$ is a nondecreasing self-map on $X, F: X \rightarrow 2^{X}$ is $g$-CAV and the following holds:

$$
f\left(H_{p}(F x, F y)\right) \leq \psi\left(f\left(M_{g}(x, y)\right)\right)
$$

for any $(x, y) \in \Delta_{g}$, where $f \in \digamma_{s}$ and $\psi \in \Phi$ and

$$
M_{g}(x, y)=\max \left\{p(g x, g y), p(g x, F x), p(g y, F y), \frac{p(g x, F y)+p(g y, F x)}{2}\right\} .
$$

If $X$ has the subsequential limit comparison property and $g(X)$ is closed, then $F$ and $g$ have a coincidence point. Moreover, $F$ and $g$ have a common fixed point if any one of the conditions (i)-(v) holds as in Theorem 1.

Proof Following similar arguments to those given in Theorem 1 and assuming $F$ is $g$-CAV, we obtain a sequence $\left\{g x_{n}\right\}$ whose consecutive terms are comparable, satisfy (1.2) and (1.4), and the following hold:

$$
g x_{n+1} \in F x_{n}, \quad \lim _{n \rightarrow \infty} g x_{n}=g x .
$$

Since $X$ has the subsequential limit comparison property, so $\left\{g x_{n}\right\}$ has a subsequence $\left\{g x_{n_{k}}\right\}$ whose every term is comparable to $g x$. Now, we prove $g x \in F x$. Obviously,

$$
\begin{aligned}
p\left(g x_{n_{k}+2}, F x\right) & \leq p\left(g x_{n_{k}+2}, g x_{n_{k}+1}\right)+p\left(g x_{n_{k}+1}, F x\right) \\
& \leq p\left(g x_{n_{k}+2}, g x_{n_{k}+1}\right)+\sup _{t \in F x_{n_{k}}} p(t, F x) \\
& \leq p\left(g x_{n_{k}+2}, g x_{n_{k}+1}\right)+H_{p}\left(F x_{n_{k}}, F x\right)
\end{aligned}
$$

for $k=0,1,2, \ldots$ For $\varepsilon>0$, there exists $k_{0}$ such that

$$
f\left(p\left(g x_{n_{k}+2}, g x_{n_{k}+1}\right)\right)<\varepsilon
$$

for all $k>k_{0}$. As

$$
\lim _{k \rightarrow \infty} f\left(p\left(g x_{n_{k}+2}, g x_{n_{k}+1}\right)\right)=0 .
$$

Since $g x_{n_{k}}$ is comparable to $g x$ for each $k$, therefore

$$
\begin{aligned}
f\left(p\left(g x_{n_{k}+2}, F x\right)\right) & \leq f\left(p\left(g x_{n_{k}+2}, g x_{n_{k}+1}\right)+H_{p}\left(F x_{n_{k}}, F x\right)\right) \\
& \leq f\left(p\left(g x_{n_{k}+2}, g x_{n_{k}+1}\right)\right)+f\left(H_{p}\left(F x_{n_{k}}, F x\right)\right)
\end{aligned}
$$




$$
\begin{aligned}
& \leq \psi\left(f\left(M_{g}\left(x_{n_{k}}, x\right)\right)\right)+\varepsilon \\
& <f\left(M_{g}\left(x_{n_{k}}, x\right)\right)+\varepsilon .
\end{aligned}
$$

Note that $f$ is continuous and $\lim _{k \rightarrow \infty} p\left(g x_{n_{k}}, F x\right)=p(g x, F x)$. We obtain, by letting $k \rightarrow \infty$,

$$
f(p(g x, F x))<f(p(g x, F x))+\varepsilon .
$$

This implies that $p(g x, F x)=0$, so we have $g x \in F x$. By similar arguments to those in Theorem 1, we can show the existence of a common fixed point.

\section{Competing interests}

The author declares that they have no competing interests.

Received: 22 August 2012 Accepted: 6 February 2013 Published: 22 February 2013

\section{References}

1. Nadler, SB: Multi-valued contraction mappings. Pac. J. Math. 20(2), 475-488 (1969)

2. Dhage, BC: Hybrid fixed point theory for strictly monotone increasing multivalued mappings with applications. Comput. Math. Appl. 53, 803-824 (2007)

3. Dhage, BC: A general multivalued hybrid fixed point theorem and perturbed differential inclusions. Nonlinear Anal. TMA 64, 2747-2772 (2006)

4. Shen, M, Hong, $\mathrm{SH}$ : Common fixed points for generalized contractive multivalued operators in complete metric spaces. Appl. Math. Lett. 22, 1864-1869 (2009)

5. Ladde, GS, Lakshmikantham, V, Vatsala, AS: Monotone Iterative Techniques for Nonlinear Differential Equations. Pitman, New York (1985)

6. Chang, SS, Ma, YH: Coupled fixed points for mixed monotone condensing operators and an existence theorem of the solutions for a class of function equations arising in dynamic programming. J. Math. Anal. Appl. 160, 468-479 (1991)

7. Hong, SH: Fixed points of multivalued operators in ordered metric spaces with applications. Nonlinear Anal. 72 , 3929-3942 (2010)

8. Abbas, $M$, Erduran, $A$ : Common fixed point of $g$-approximative multivalued mapping in partially ordered metric space. Filomat (Accepted)

9. Jungck, G, Rhoades, BE: Fixed points for set valued functions without continuity. Indian J. Pure Appl. Math. 29, 227-238 (1998)

10. Zhang, $X$ : Common fixed point theorems for some new generalized contractive type mappings. J. Math. Anal. Appl. 333, 780-786 (2007)

11. Matthews, SG: Partial metric topology. In: Proc. 8th Summer Conference on General Topology and Applications. Ann. New York Acad. Sci., vol. 728, pp. 183-197 (1994)

12. Altun, I, Simsek, H: Some fixed point theorems on dualistic partial metric spaces. J. Adv. Math. Stud. 1, 1-8 (2008)

13. Altun, I, Sola, F, Simsek, H: Generalized contractions on partial metric spaces. Topol. Appl. 157, 2778-2785 (2010)

14. Aydi, H, Abbas, M, Vetro, C: Partial Hausdorff metric and Nadler's fixed point theorem on partial metric spaces. Topol. Appl. 159, 3234-3242 (2012)

doi:10.1186/1687-1812-2013-36

Cite this article as: Erduran: Common fixed point of $g$-approximative multivalued mapping in ordered partial metric space. Fixed Point Theory and Applications 2013 2013:36.

\section{Submit your manuscript to a SpringerOpen ${ }^{\ominus}$ journal and benefit from:}

- Convenient online submission

- Rigorous peer review

- Immediate publication on acceptance

Open access: articles freely available online

- High visibility within the field

- Retaining the copyright to your article 